\title{
Discussion Group 6 - Building resource accessibility in developing countries
}

Chair: Ian Mitchell (New Zealand)

Particpants: Kersti Hjertqvist (Sweden), J. R. Isaac (India), John Manganye (Zimbabwe), Nathan Matemera (Zimbabwe), Tendeka Matatu (Zimbabwe), Edmore Muchinapo (Zimbabwe), Paul Nleya (Botswana), John Nyamhotsi (Zimbabwe), Alex Tigere (Zimbabwe)

\section{PROBLEM DEFINITION}

The first step is to clarify the problem.

\subsection{Hardware resources}

The hardware resources required are:

- PCs, network server, router for connection to the Internet and, possibly later, network computers.

\subsection{Software resources}

The software resources required are:

- Courseware - Office suite, database package, statistics package, Internet browser.

Some software will need to be localised, especially for appropriate teaching examples and, for some countries, languages.

Note: Courseware should not be 'the book' on multimedia. It should be purposedesigned with a sound pedagogical structure.

\subsection{Human resources}

Trained principals, teachers and technicians will be required. Vocational trainers with work experience will be needed for training practitioners. Courseware developers (subject experts, instructional designers, developers and multimedia designers) will be needed for local courseware development. 


\subsection{Infrastructural resources}

Fundamental requirements are:

- A stable electrical supply, telephone lines with adequate bandwidth and transport to teacher training venues.

2

\section{ACCESSIBILITY}

For accessibility the resources must be available, operative with persons knowing how to use them and with confidence to do so. For software to be shared it must be portable and issues of intellectual property rights, language, culture and political will must be addressed. A major inhibitor may be cost. More important than software is the sharing of knowledge, expertise and competency.

\section{GOAL RE-STATED}

Every child in all developing countries has fun learning 'hands-on' with operative computers running relevant software.

\subsection{Short-term goals}

Such a goal is ambitious, demanding high levels of resources, efforts and time. Therefore, we focused on shorter-term objectives which might be satisfied within one year and at less than SUS 250000.

A comprehensive national policy must be developed. It must reflect national goals and aspirations but clearly indicate what principals, teachers and parents may do in the school system with the encouragement of governments.

An enthusiastic community will make things happen for their children. The community may be engaged by being shown computers being effectively used. The community may then choose which initiatives to support and then commit to contribute resources to the school by fund-raising but also by providing support. An example is the Net Day projects in the USA, where the community helps 'wire' the school.

Some examples are discussed below.

\subsection{Teacher training}

A donor may provide a computer laboratory for teacher training. As well as hardware and software the donor may provide an expert trainer. The recipient may provide several persons to become trainers and a technician. 
Teachers who are about to get computers in their schools should attend a course of 3-5 days. Subsequently the trainers should visit the teachers about two weeks later and again two months later. Therefore the trainers not only give the teachers further support but also learn what are the practical problems that teachers face in the schools.

In subsequent years the aid recipient nationals can take over.

\subsection{Mobile laboratory}

A mobile laboratory can be installed in a suitable truck. An expert trainer and a technician/driver should travel with the truck. The truck should contain a supplementary generator allowing the laboratory to operate where there is no electrical supply.

The truck might spend a week with the community. The local primary and secondary schools would schedule time for their pupils to use the facilities. Special courses might be run for those needing vocational training. Small business people in the community might be introduced to computers. The laboratory could be used 12 hours per day.

The community awareness of computing in education would be raised. The community could then decide where computers might first be introduced and what they will do to help or encourage the teachers and the school.

\subsection{Internet gateway}

An Internet service provider might be contracted to run a school's gateway server on behalf of the school system. The server might have substantial cache memory (say $100 \mathrm{~GB}$ ) so that information fetched from international sources can be held locally and reduce the communications costs for subsequent accesses. Such a computer would cost less than \$US 20000.

The server could have positive or negative stop lists so that pornography and other unsuitable materials can be suppressed. Further caching servers could be placed in each school district to reduce toll loads. Finally, a school should have a network server and this, too, could cache local accesses. Such software could be 'off the shelf'.

\section{A note on the pedagogical uses of the Internet}

The pupils should seek whatever information they are interested in on the World Wide Web and increase their knowledge on issues they have chosen themselves. Pupils can collaborate with pupils in their own country or in other countries. Teachers can collaborate with other teachers in their own country or in other countries. Teachers can collect teaching materials that other teachers or institutions have put on the Internet, thus always having up-to-date information. IT teacher training can be followed on the Internet in one's own school instead of traveling to the nearest town.

Pupils can create their own learning materials, which can be used by other pupils when they study. Pupils can produce the learning materials in multimedia presentations. They can quickly learn how to handle a scanner, video capture 
facilities, digital cameras or a color printer in order to produce multimedia materials.

\subsection{Funding teachers' computers}

Teachers may be provided with loans, or with finance or leasing arrangements so that they can obtain a computer and gain experience and confidence in using the computer. Alternatively, computers may be funded and placed in public libraries, including traveling libraries, so that more people can become familiar with them. Old computers from business may be given to schools provided they are suitable.

\subsection{Seeking donors}

To gain donors for a project, a well-prepared proposal must be put forward. Donors must be identified who are interested in the education and capacity-building areas. Most donors operate with governments and therefore you must seek the assistance of your government policy advisers who have this responsibility.

\subsection{Government commitment}

The government must give a clear commitment to support the use of computers in schools and in vocational training arenas in the use of computers at both user and professional levels. The government should identify the initiatives they will support and clearly commit to donors that successful and proven projects will be replicated and multiplied. A similar commitment will be needed to support community projects. 